\title{
Wiener Integration for Quantum Systems: A Unified Approach to the Feynman-Kac formula *
}

\author{
Bernhard Bodmann, Hajo Leschke and Simone Warzel \\ Institut für Theoretische Physik \\ Universität Erlangen-Nürnberg \\ Staudtstraße 7, D-91058 Erlangen, Germany
}

\begin{abstract}
A generalized Feynman-Kac formula based on the Wiener measure is presented. Within the setting of a quantum particle in an electromagnetic field it yields the standard Feynman-Kac formula for the corresponding Schrödinger semigroup. In this case rigorous criteria for its validity are compiled. Finally, phase-space path-integral representations for more general quantum Hamiltonians are derived. These representations rely on a generalized Lie-Trotter formula which takes care of the operator-ordering multiplicity, but in general is not related to a path measure.
\end{abstract}

Actually, in the Wiener integral the things are much simpler. [46]

\section{The Feynman-Kac formula, revisited}

More than seventy years of Wiener's path integration have been most gratifying for both mathematicians and theoretical physicists. Originally constructed as a mathematical model for the phenomenon of Brownian motion, it nowadays plays a major rôle also in polymer and quantum physics, and still is fundamental to the theory of general stochastic processes [39]. The importance of Wiener's measure [45] for quantum physics became clear soon after Feynman's stimulating paper [16] when Kac [24, 25] identified it as the key to a probabilistic representation of Schrödinger semigroups, these days called the Feynman-Kac formula [42, 20, 40].

In this section we intend to survey essentials of this formula and some of its relatives. In doing so, we leave aside most technicalities and subtleties which we believe to be of secondary importance for applications, in particular when they tend to obscure the simplicity of the underlying ideas.

${ }^{*}$ Partially based on a plenary lecture given by H. L. at the international conference on path integrals, Dubna, Russia, May 27-31, 1996; in: Path integrals: Dubna '96, eds. V. S. Yarunin and M. A. Smondyrev, JINR E96-321, ISBN: 5-85165-451-1, Dubna 1996, pp. 95-106. 


\subsection{The Wiener measure and stochastic integrals}

The standard Wiener measure $D w$ is a certain probability distribution on the space of continuous paths $\left\{w: \mathbb{R}_{+} \rightarrow \mathbb{R}^{d}, s \mapsto w(s)\right\}$ from the positive halfline $\mathbb{R}_{+}:=\left[0, \infty\left[\right.\right.$ into $d$-dimensional Euclidean space $\mathbb{R}^{d}$ which start at the origin, $w(0)=0$. It is uniquely determined and well-defined by the following properties:

i) $D w$ is a Gaussian probability measure.

ii) Its first and second moments are

$$
\int D w w_{j}(s)=0, \quad \int D w w_{j}(r) w_{k}(s)=\delta_{j k} \min (r, s) .
$$

Here and in the following indices $j, k, \ldots$ denumerate the components of $d$-component quantities, $x \cdot y:=\sum_{j=1}^{d} x_{j} y_{j}$ denotes the standard scalar product of two such quantities, and the usual convention $x^{2}:=x \cdot x$ is adopted. Kronecker's delta in (ii) expresses the stochastic independence of different components of $w$. A one-line characterization of $D w$, equivalent to i) and ii), is given by its (functional) Fourier transform

$$
\int D w \exp \left\{-i \int_{0}^{\infty} d s w(s) \cdot f(s)\right\}=\exp \left\{-\frac{1}{2} \int_{0}^{\infty} d r \int_{0}^{\infty} d s \min (r, s) f(r) \cdot f(s)\right\}
$$

where $f: \mathbb{R}_{+} \rightarrow \mathbb{R}^{d}$ is any $d$-component function with compact support in the half-line $\mathbb{R}_{+}$.

Whenever the Wiener measure $D w=\prod_{1 \leq j \leq d} D w_{j}$ is applicable, its advantages over lattice or time-slicing prescriptions for path integration can hardly be overestimated. Since $D w$ is a positive, countably additive and normalized measure, the powerful machinery of general integration and probability theory [29, (7] is at hand for the computation or estimation of Wiener integrals $\int D w F(w)$, where $F$ is a functional of the paths. The importance of $D w$ for integration in infinite-dimensional path space $\left(\mathbb{R}^{d}\right)^{\mathbb{R}_{+}}$is similar to that of Lebesgue's measure $d x=\prod_{1 \leq j \leq d} d x_{j}$ for 'ordinary' integration in $\mathbb{R}^{d}$.

For an intuitive understanding of $D w$ it is sometimes helpful to think of it as an infinite-dimensional Lebesgue measure with Gaussian weight

$$
\prod_{\substack{0<s \\ 1 \leq j \leq d}}\left(\frac{d w_{j}(s)}{\sqrt{2 \pi \Delta s}} \exp \left\{-\frac{\Delta s}{2} \dot{w}_{j}(s)^{2}\right\}\right)
$$

in the limit $\Delta s \downarrow 0$. Strictly speaking, expression (3) is meaningless because there is no translational invariant measure in infinite dimensions. In addition, Wiener paths almost surely have no time derivative $\dot{w}:=\frac{d}{d s} w$ for any $s$ and hence are not of bounded variation on any compact interval $[0, t]$, see e.g. [7]. Nevertheless, the concept of stochastic integrals allows to give precise meaning in a suitable probabilistic sense to (line) integrals of the form

$$
\int_{0}^{t} d s \dot{w}(s) \cdot g(w(s), s), \quad t \geq 0
$$

for a wide class of functions $g: \mathbb{R}^{d} \times \mathbb{R}_{+} \rightarrow \mathbb{R}^{d}$. In principle, there is a huge 
multiplicity of rigorously definable candidates [39, p. 136] for (4). As a rule however, literature [5, 33, 39] discusses at most a one-parameter subclass of stochastic integrals, denoted by us as

$$
\text { @ } \int_{0}^{t} d w(s) \cdot g(w(s), s), \quad \alpha \in[0,1] .
$$

While probabilists usually use $\alpha=0$ corresponding to Itô's original proposal, we will here follow Stratonovich in choosing $\alpha=1 / 2$ for the interpretation of (雨), so that the rules of ordinary calculus in the sense of Newton and Leibniz formally apply. By the conversion formula [33, p. 113] [39, p. 136]

$$
\int_{0}^{t} d s \dot{w}(s) \cdot g(w(s), s)=\propto \int_{0}^{t} d w(s) \cdot g(w(s), s)+\left(\frac{1}{2}-\alpha\right) \int_{0}^{t} d s(\nabla \cdot g)(w(s), s)
$$

the Stratonovich stochastic integral is related to the $\alpha$-stochastic integral. Note that the second integral on the right-hand side containing the divergence of $g$ is understood in the Lebesgue sense because of the continuity of Wiener paths. Stochastic integrals can also be defined for functions $(w, s) \mapsto g(w, s)$ which in their first argument may depend on the whole history $\{w(r)\}_{r \leq s}$ of the path up to the time value of the second argument [5, 33, 39]. Such non-anticipating functions might take values in $\mathbb{R}^{d}$, in a Hilbert space or even in a set of Hilbert-space operators [20].

As a simple application of the preceding discussion one obtains

$$
\int D w \exp \left\{-i \int_{0}^{\infty} d s \dot{w}(s) \cdot f(s)\right\}=\exp \left\{-\frac{1}{2} \int_{0}^{\infty} d s f(s)^{2}\right\}
$$

for compactly supported $f$. This follows from (formal) integration by parts, equation (2) and the representation $\delta(r-s)=\frac{\partial^{2}}{\partial r \partial s} \min (r, s)$ of Dirac's delta function. In analogy to (2), equation (7) may serve as a one-line characterization of $d$-component Gaussian white noise.

\subsection{A generalization of the Feynman-Kac formula}

The main purpose of this subsection is to extend equation (7) to certain operatorvalued functions $f$. Accordingly, let $\widehat{A}=\left(\widehat{A}_{1}, \ldots, \widehat{A}_{d}\right)$ be a $d$-component operator acting on some separable Hilbert space $\mathcal{H}$. Moreover, let $\widehat{B}$ be another operator and $\hat{1}$ the identity on $\mathcal{H}$. For notational simplicity we will assume that $\widehat{A}$ and $\widehat{B}$ are time-independent, the extension to the time-dependent case being straightforward. Finally, let $\widehat{T}_{t}(w)$ be the operator solving the linear stochastic integral equation

$$
\widehat{T}_{t}=\hat{1}-i \int_{0}^{t} d s \dot{w}(s) \cdot \widehat{A} \widehat{T}_{s}-\widehat{B} \int_{0}^{t} d s \widehat{T}_{s}
$$

which is the precise formulation of the linear (Stratonovich) stochastic differential equation

$$
\frac{d}{d t} \widehat{T}_{t}=-i(\dot{w}(t) \cdot \widehat{A}-i \widehat{B}) \widehat{T}_{t}, \quad \widehat{T}_{0}=\hat{1} .
$$

In analogy to quantum dynamics with explicitly time-dependent Hamiltonians the 
solution $\widehat{T}_{t}(w)$ can be obtained by iterating (8)

$$
\begin{aligned}
\widehat{T}_{t}(w)= & \hat{1}+\sum_{n=1}^{\infty}(-i)^{n} \int_{0}^{t} d s_{n}\left(\dot{w}\left(s_{n}\right) \cdot \widehat{A}-i \widehat{B}\right) \int_{0}^{s_{n}} d s_{n-1} \\
& \quad \times\left(\dot{w}\left(s_{n-1}\right) \cdot \widehat{A}-i \widehat{B}\right) \cdots \int_{0}^{s_{2}} d s_{1}\left(\dot{w}\left(s_{1}\right) \cdot \widehat{A}-i \widehat{B}\right) \\
= & \overleftarrow{\exp }\left\{-i \int_{0}^{t} d s(\dot{w}(s) \cdot \widehat{A}-i \widehat{B})\right\}
\end{aligned}
$$

which defines Dyson's time--ordered exponential function $\overleftarrow{\exp }$, also known as product integral.

Now we are prepared to state a generalized Feynman-Kac formula

$$
\int D w \widehat{T}_{t}(w)=\exp \left\{-t\left(\frac{1}{2} \widehat{A}^{2}+\widehat{B}\right)\right\} .
$$

As we will see in the next subsection, the standard Feynman-Kac formula follows by a suitable choice of $\mathcal{H}, \widehat{A}$ and $\widehat{B}$. A heuristic argument for the validity of (12) goes as follows: In the presence of time-ordering, $\widehat{B}$ and all components of $\widehat{A}$ may be treated as pairwise commuting [17, 28], so that a formal application of (7) to (10) with $f(s)=\Theta(t-s) \widehat{A}$ gives the desired result. Here $\Theta$ stands for Heaviside's unit-step function.

A more convincing argument for (12) proceeds in two steps. First consider the case of vanishing $\widehat{B}$. Wiener integrating both sides of (8) and supposing

$$
\left\langle\int_{0}^{t} d s \dot{w}_{j}(s) \widehat{T}_{s}\right\rangle=-\frac{i}{2} \widehat{A}_{j}\left\langle\int_{0}^{t} d s \widehat{T}_{s}\right\rangle
$$

where for convenience the notation $\langle\cdot\rangle:=\int D w(\cdot)$ is introduced, yields the integral equation

$$
\left\langle\widehat{T}_{t}\right\rangle=\hat{1}-\frac{1}{2} \widehat{A}^{2} \int_{0}^{t} d s\left\langle\widehat{T}_{s}\right\rangle
$$

which, in turn, gives $\left\langle\widehat{T}_{t}\right\rangle=\exp \left\{-\frac{t}{2} \widehat{A}^{2}\right\}$. Equation (13) follows from integration by parts with respect to $D w$ using its Gaussian nature (cf. [41, p. 32 and solution of exercise 1.8.22]) and the convention $\Theta(0):=1 / 2$ corresponding to the Stratonovich interpretation. For a rigorous proof of (13) one should use Malliavin's stochastic variational calculus [37, 32]. Clearly, if the components of $\widehat{A}$ commute, time ordering can be dropped, so that the result is obtained directly from $\widehat{T}_{t}(w)=\exp (-i w(t) \cdot \widehat{A})$ and (目).

The case $\widehat{B} \neq \widehat{0}$ will now be treated in the second step by a perturbative argument in the spirit of Kac's original proof for the standard Feynman-Kac formula (see [25, 42, 40]). For this purpose, we define

$$
\widehat{U}_{t}(w):=\overleftarrow{\exp }\left\{-i \int_{0}^{t} d s \dot{w}(s) \cdot \widehat{A}\right\}
$$

and 


$$
\begin{aligned}
\widehat{S}_{t}(w) & :=\overleftarrow{\exp }\left\{-\int_{0}^{t} d s\left(\widehat{U}_{s}(w)\right)^{-1} \widehat{B} \widehat{U}_{s}(w)\right\} \\
& =\hat{1}-\int_{0}^{t} d s\left(\widehat{U}_{s}(w)\right)^{-1} \widehat{B} \widehat{U}_{s}(w) \widehat{S}_{s}(w) .
\end{aligned}
$$

Then, according to standard time-dependent perturbation theory

$$
\widehat{T}_{t}=\widehat{U}_{t} \widehat{S}_{t}=\widehat{U}_{t}-\int_{0}^{t} d s \widehat{U}_{t} \widehat{U}_{s}^{-1} \widehat{B} \widehat{T}_{s}
$$

We now Wiener integrate both sides, assume the identity

$$
\left\langle\widehat{U}_{t} \widehat{U}_{s}^{-1} \widehat{B} \widehat{T}_{s}\right\rangle=\left\langle\widehat{U}_{t-s}\right\rangle \widehat{B}\left\langle\widehat{T}_{s}\right\rangle
$$

and recall $\left\langle\widehat{U}_{t}\right\rangle=\exp \left\{-\frac{t}{2} \widehat{A}^{2}\right\}$ from the first step to obtain the Duhamel-type of integral equation

$$
\left\langle\widehat{T}_{t}\right\rangle=\exp \left\{-\frac{t}{2} \widehat{A}^{2}\right\}-\int_{0}^{t} d s \exp \left\{-\frac{t-s}{2} \widehat{A}^{2}\right\} \widehat{B}\left\langle\widehat{T}_{s}\right\rangle .
$$

This implies the initial-value problem

$$
\frac{d}{d t}\left\langle\widehat{T}_{t}\right\rangle=-\left(\frac{1}{2} \widehat{A}^{2}+\widehat{B}\right)\left\langle\widehat{T}_{t}\right\rangle, \quad\left\langle\widehat{T}_{0}\right\rangle=\hat{1}
$$

which finally gives (12). The claimed stochastic independence underlying (19) is basically due to the Markov property of $D w$ and the fact that Wiener paths start continually afresh 42, 7.

We conclude this subsection with four remarks:

- Clearly, the Markov property of the Wiener measure $D w$ is responsible for the emergence of a one-parameter operator semigroup on the right-hand side of the generalized Feynman-Kac formula (12). The Gaussian nature of $D w$ causes (the negative of) its generator $\frac{1}{2} \widehat{A}^{2}+\widehat{B}$ to be quadratic in $\widehat{A}$. If $\widehat{A}$ and $\widehat{B}$ are Hermitian, the left-hand side manifestly is, because $D w$ does not change under reflection $w(s) \mapsto-w(s)$.

- Conversely, reading (12) from right to left may be viewed as uncompleting squares of operators in an exponent by Gaussian linearization. It is therefore closely related to the so-called Hubbard-Stratonovich trick, widely used in different branches of theoretical physics. For applications to certain manybody systems see the surveys [34, 27] and references therein. To our knowledge, so far mainly the case of commuting $\widehat{A}$-components has been considered in this context. There is also a noteworthy similarity to Feynman's disentangling formalism [17, 35].

- A simple corollary of (12) for $d=2$, namely

$$
\begin{aligned}
& \int D w \overleftarrow{\exp }\left\{-i \int_{0}^{t} d s\left[\left(\dot{w}_{1}(s)+i \dot{w}_{2}(s)\right) \widehat{A}_{+}+\left(\dot{w}_{1}(s)-i \dot{w}_{2}(s)\right) \widehat{A}_{-}-i \widehat{B}\right]\right\} \\
& =\exp \left\{-t\left(\widehat{A}_{+} \widehat{A}_{-}+\widehat{A}_{-} \widehat{A}_{+}+\widehat{B}\right)\right\}
\end{aligned}
$$


follows from the non-commutative extension

$$
\widehat{A}_{+} \widehat{A}_{-}+\widehat{A}_{-} \widehat{A}_{+}=\frac{1}{2}\left(\widehat{A}_{+}+\widehat{A}_{-}\right)^{2}-\frac{1}{2}\left(\widehat{A}_{+}-\widehat{A}_{-}\right)^{2}
$$

of the binomial formula for a pair of operators $\widehat{A}_{+}$and $\widehat{A}_{-}$. This shows that one can also linearize symmetrized products of two, in general non-commuting operators.

- In case of an infinite-dimensional Hilbert space $\mathcal{H}$ the arguments and computations in our derivation are rather formal, unless one specifies a class of admissible operators $\widehat{A}$ and $\widehat{B}$ which is sufficiently wide to be of interest for applications. In particular, domain questions require additional efforts. See, for example, subsection 1.4 below.

\subsection{The standard Feynman-Kac formula}

In this subsection we specialize to the usual setting for non-relativistic quantum mechanics of an electrically charged point mass in $\mathbb{R}^{d}$ without internal degrees of freedom. Consequently, let $L^{2}\left(\mathbb{R}^{d}\right)$ be the Hilbert space of complex-valued functions $\psi: \mathbb{R}^{d} \rightarrow \mathbb{C}, q \mapsto \psi(q)$, which are square-integrable with respect to the Lebesgue measure $d q$. Consider now a vector potential $a: \mathbb{R}^{d} \rightarrow \mathbb{R}^{d}$ and a scalar potential $v$ : $\mathbb{R}^{d} \rightarrow \mathbb{R}$, both sufficiently well-behaved. They give rise to a standard Hamiltonian, or Schrödinger operator, $\widehat{H}:=\frac{1}{2}(\hat{p}-a(\hat{q}))^{2}+v(\hat{q})$ on $L^{2}\left(\mathbb{R}^{d}\right)$ with magnetic field $b_{j k}:=\frac{\partial a_{j}}{\partial q_{k}}-\frac{\partial a_{k}}{\partial q_{j}}$. Here $\hat{p}$ and $\hat{q}$ denote the usual self-adjoint momentum and position operators. For notational simplicity, we choose units such that Planck's constant $\hbar$, the mass and the charge of the particle are all unity.

Then the standard Feynman-Kac formula with a vector potential reads

$$
\mathrm{e}^{-t \widehat{H}}=\int D w \mathrm{e}^{-i w(t) \cdot \hat{p}} \exp \left\{i \int_{0}^{t} d s \dot{w}(s) \cdot a(\hat{q}+w(s))-\int_{0}^{t} d s v(\hat{q}+w(s))\right\}
$$

In fact, it is a special case of (12) for the choice $\mathcal{H}=L^{2}\left(\mathbb{R}^{d}\right), \hat{A}=\hat{p}-a(\hat{q})$ and $\widehat{B}=v(\hat{q})$. This follows from the first equation in (18) and the identity

$$
\begin{aligned}
\widehat{U}_{t}(w) & =\overleftarrow{\exp }\left\{-i \int_{0}^{t} d s \dot{w}(s) \cdot(\hat{p}-a(\hat{q}))\right\} \\
& =\mathrm{e}^{-i w(t) \cdot \hat{p}} \exp \left\{i \int_{0}^{t} d s \dot{w}(s) \cdot a(\hat{q}+w(s))\right\}
\end{aligned}
$$

which is again understood with the help of time-dependent perturbation theory in combination with the fact that the time-dependent operator components

$$
\mathrm{e}^{i w(s) \cdot \hat{p}} a_{j}(\hat{q}) \mathrm{e}^{-i w(s) \cdot \hat{p}}=a_{j}(\hat{q}+w(s))
$$

commute for all values of $j$ and $s$. The specialization is completed by observing a similar shift-identity for the scalar potential in $\widehat{S}_{t}(w)$.

Several remarks apply: 
- The Wiener integral has managed to completely disentangle the non-commuting operators $\hat{p}$ and $\hat{q}$ in the Schrödinger semigroup $\exp (-t \widehat{H})$. The standard Feynman-Kac formula in its basis-independent version (24) has been presented before (cf. e.g. [19, 1, 9]). Apart from mathematical subtleties, this abstract way of looking at the Feynman-Kac formula extracts its essential ingredients and is often a convenient starting point for further manipulations.

- The Stratonovich stochastic integral in (24) may be replaced by the corresponding $\alpha$-stochastic integral using the conversion formula (6). The resulting formula might be called the Feynman-Kac- $\alpha$ formula extending a terminology introduced by Simon [42]. The correct unitary transformation $\mathrm{e}^{-t \widehat{H}} \mapsto \mathrm{e}^{i \chi(\hat{q})} \mathrm{e}^{-t \widehat{H}} \mathrm{e}^{-i \chi(\hat{q})}$ under a change of gauge $a \mapsto a+\nabla \chi$ is most easily obtained from (24) by using the rules of ordinary calculus.

- By applying both sides of (24) to a wave function $\psi \in L^{2}\left(\mathbb{R}^{d}\right)$ it is straightforward to derive the usual probabilistic expression for its image $\exp (-t \widehat{H}) \psi$ under the Schrödinger semigroup (cf. [42, eqs. (6.8) and (15.1)])

$$
\begin{aligned}
&\left(\mathrm{e}^{-t \widehat{H}} \psi\right)(q)=\int D w \exp \left\{-i \int_{0}^{t} d s \dot{w}(s) \cdot a(q+w(s))\right\} \\
& \times \exp \left\{-\int_{0}^{t} d s v(q+w(s))\right\} \psi(q+w(t)) .
\end{aligned}
$$

This can be rewritten as

$$
\left(\mathrm{e}^{-t \widehat{H}} \psi\right)(q)=\int d q^{\prime}\left\langle q\left|\mathrm{e}^{-t \widehat{H}}\right| q^{\prime}\right\rangle \psi\left(q^{\prime}\right)
$$

where the Euclidean propagator, that is, the position representation of the semigroup, is given by

$$
\begin{gathered}
\left\langle q\left|\mathrm{e}^{-t \widehat{H}}\right| q^{\prime}\right\rangle=\int D w \delta\left(w(t)+q-q^{\prime}\right) \exp \left\{-i \int_{0}^{t} d s \dot{w}(s) \cdot a(q+w(s))\right\} \\
\times \exp \left\{-\int_{0}^{t} d s v(q+w(s))\right\} .
\end{gathered}
$$

Here, due to the presence of the delta function, effectively only those Wiener paths contribute which pass through $q^{\prime}-q$ at time $t$. One may also integrate over the rigidly shifted paths $s \mapsto w(s)+q$ which start at $q$ and pass through $q^{\prime}$ at time $t$.

- As a final point in this subsection, we mention a close relative of (28), the probabilistic representation of the unitary time evolution

$$
\begin{aligned}
\left(\mathrm{e}^{-i t \widehat{H}} \psi\right)(q)=\int & D w \exp \left\{-i \sqrt{i} \int_{0}^{t} d s \dot{w}(s) \cdot a(q+\sqrt{i} w(s))\right\} \\
& \times \exp \left\{-i \int_{0}^{t} d s v(q+\sqrt{i} w(s))\right\} \psi(q+\sqrt{i} w(t)) .
\end{aligned}
$$

It is obtained analogously to (24) and (28) by choosing $\widehat{A}=\sqrt{i}(\hat{p}-a(\hat{q}))$ and $\widehat{B}=i v(\hat{q})$ in (12). Its validity obviously requires some analyticity assumptions on the potentials and the (initial) wavefunction. For rigorous discussions of (31) (in the case $a=0$ ), see [21, 6] and the book [22] on a related theme. 


\subsection{On the validity of the standard Feynman-Kac formula}

Equations (28) and (30) are the most popular versions of the Feynman-Kac formula. It is therefore fortunate that there is a wealth of information about the conditions under which they are rigorously valid. We take the opportunity to recall some rather weak assumptions on the potentials $a$ and $v$ which are sufficient [42, 43, 3, 12, 10]. To this end, let $L_{l o c}^{p}\left(\mathbb{R}^{d}\right), p \in[1, \infty[$, denote the space of complex-valued functions $\varphi$ : $\mathbb{R}^{d} \rightarrow \mathbb{C}$ with $\int_{\Lambda} d q|\varphi(q)|^{p}<\infty$ for all compact $\Lambda \subset \mathbb{R}^{d}$. Let $v_{ \pm}(q):=\sup \{0, \pm v(q)\}$ be the positive and negative part of the scalar potential $v=v_{+}-v_{-}$.

In a first step we show the existence of the Wiener integral in (28) for $a=0$ and $v_{-}$belonging to the Katô class $\mathcal{K}\left(\mathbb{R}^{d}\right)$, that is, $\lim _{t \downarrow 0} \kappa_{t}\left(v_{-}\right)=0$ where

$$
\kappa_{t}(u):=\sup _{x \in \mathbb{R}^{d}} \int D w \int_{0}^{t} d s|u(x+w(s))|=\sup _{x \in \mathbb{R}^{d}} \int_{0}^{t} d s\left(\mathrm{e}^{-s \hat{p}^{2} / 2}|u|\right)(x) .
$$

Due to the Markov property of $D w$ one may assume without loss of generality $t>0$ sufficiently small, so that $\kappa_{t}\left(v_{-}\right)<1 / 2$. Then the following estimate holds

$$
\left|\int D w \exp \left\{-\int_{0}^{t} d s v(q+w(s))\right\} \psi(q+w(t))\right|^{2} \leq \frac{\int d x|\psi(x)|^{2}}{(2 \pi t)^{d / 2}\left(1-2 \kappa_{t}\left(v_{-}\right)\right)} .
$$

Its proof starts from employing the Cauchy-Schwarz-Bunyakovski inequality

$$
\left|\left\langle M_{t}(q, v) \psi(q+w(t))\right\rangle\right|^{2} \leq\left\langle M_{t}(q, 2 v)\right\rangle\left\langle|\psi(q+w(t))|^{2}\right\rangle
$$

with respect to $D w$, where we have introduced the (multiplicative) functional

$$
M_{t}(q, v):=\exp \left\{-\int_{0}^{t} d s v(q+w(s))\right\} .
$$

While the second factor on the right-hand side of (34) is easily seen not to exceed $\int d x|\psi(x)|^{2} /(2 \pi t)^{d / 2}<\infty$, the first factor is estimated using $-v \leq v_{-}$and Khas'minskii's Lemma [26, 33, 43] $\left\langle M_{t}\left(q,-v_{-}\right)\right\rangle \leq\left(1-\kappa_{t}\left(v_{-}\right)\right)^{-1}$.

Now turning on the vector potential $a$, the conditions $a^{2}, \nabla \cdot a \in L_{l o c}^{1}\left(\mathbb{R}^{d}\right)$ are sufficient for the existence of the Stratonovich stochastic integral in (28) (and hence of the corresponding $\alpha$-stochastic integral for arbitrary $\alpha$ ). Since it appears there as the phase of a complex number of absolute value one, the Wiener integral in (28) remains bounded from above by the right-hand side of (33). In other words, the diamagnetic inequality [42] has extended (33) to non-vanishing $a$.

In order to guarantee equality (28), at least for Lebesgue-every $q$, one needs a condition on $v_{+}$, in addition to the above assumptions on $a$ and $v_{-}$. For example, if $v_{+} \in L_{l o c}^{1}\left(\mathbb{R}^{d}\right)$ the Hamiltonian $\widehat{H}$ can be precisely defined in the sense of quadratic forms as a self-adjoint operator on $L^{2}\left(\mathbb{R}^{d}\right)$ bounded from below and (28) holds. Under the stronger assumptions $v_{-} \in \mathcal{K}\left(\mathbb{R}^{d}\right) \cap L_{l o c}^{2}\left(\mathbb{R}^{d}\right)$ and $v_{+}, a^{2}, \nabla \cdot a \in L_{l o c}^{2}\left(\mathbb{R}^{d}\right)$ the space of arbitrarily often differentiable complex-valued functions with compact support, $C_{0}^{\infty}\left(\mathbb{R}^{d}\right)$, can even be identified as an operator domain of essential selfadjointness. 
Pointwise validity of (28) is assured by the assumptions $v_{-} \in \mathcal{K}\left(\mathbb{R}^{d}\right)$ and $v_{+}, a^{2}$, $\nabla \cdot a \in \mathcal{K}_{\text {loc }}\left(\mathbb{R}^{d}\right) \subset L_{\text {loc }}^{1}\left(\mathbb{R}^{d}\right)$, where by definition $u \in \mathcal{K}_{\text {loc }}\left(\mathbb{R}^{d}\right)$ if $u \varphi \in \mathcal{K}\left(\mathbb{R}^{d}\right)$ for all $\varphi \in C_{0}^{\infty}\left(\mathbb{R}^{d}\right)$. Under these assumptions the semigroup $\exp (-t \widehat{H})$ maps $L^{2}\left(\mathbb{R}^{d}\right)$ into its subspace of functions which are continuous (and bounded). As a consequence, both sides in (28) are continuous and hence coincide for all $q$. Even more, the semigroup possesses then for each $t>0$ an integral kernel $\left(q, q^{\prime}\right) \mapsto\left\langle q|\exp (-t \widehat{H})| q^{\prime}\right\rangle$ which is jointly continuous in $\left(t, q, q^{\prime}\right)$ for $t>0$ and $q, q^{\prime} \in \mathbb{R}^{d}$. It can be expressed by the right-hand side of (30), provided the delta function is understood in the sense of Donsker or Hida (see e.g. [22]), or the integration $\int D w \delta\left(w(t)+q-q^{\prime}\right)(\cdot)$ is interpreted as a short-hand for averaging with respect to the Brownian bridge 42, 40, 10] from $(0, q)$ to $\left(t, q^{\prime}\right)$.

An important example of a scalar potential, for which (28) holds pointwise and the right-hand side of (30) defines the corresponding continuous integral kernel, is the inverse-distance potential $v(q)=-\gamma /|q|, \gamma \in \mathbb{R}$, for $d \geq 2$. Complications in the case $d=1$ are treated in $[18$.

\section{Phase-space path integrals for non-standard Hamiltonians}

Phase-space path integrals serve to provide a generalization of the standard Feynman-Kac formula to non-standard Hamiltonians on $L^{2}\left(\mathbb{R}^{d}\right)$, that is, Hamiltonians more general than $\widehat{H}=\frac{1}{2}(\hat{p}-a(\hat{q}))^{2}+v(\hat{q})$. This idea dates back to Feynman [17] and is still used in quantum physics (see, e. g. [44, Ch. 9]). Any attempt to put it on safer grounds has to face the problem that there is no unique correspondence between Hilbert-space operators and phase-space functions. Until now the most popular method to give meaning to Feynman's heuristic idea of phase-space path integrals employs lattice or time-slicing prescriptions. Since the seventies it is wellknown 14, 14, 31, 8, 30, 38 that this method reflects the above non-uniqueness by a multiplicity of prescriptions, each linked to a particular way of choosing the order for the factors in a product of non-commuting operators. In our opinion the most systematic way to keep track of this is to proceed via linear phase-space representations (also known as symbol-calculus in the theory of pseudo-differential operators) and a generalized Lie-Trotter formula. In this section we are going to briefly summarize such a procedure, restricting ourselves for clarity to certain oneparameter subclasses among phase-space representations and corresponding lattice prescriptions.

Following 31] we associate with a given Hamilton operator $\widehat{H}$ on $L^{2}\left(\mathbb{R}^{d}\right)$ a oneparameter family of symbols, that is, functions on classical phase space $\mathbb{R}^{d} \times \mathbb{R}^{d}$ defined by

$$
H_{\alpha}(p, q):=\int d x \mathrm{e}^{i p \cdot x}\langle q-(1-\alpha) x|\widehat{H}| q+\alpha x\rangle, \quad \alpha \in[0,1] .
$$

For each fixed $\alpha$ the linear mapping $\widehat{H} \mapsto H_{\alpha}$ of Hilbert-space operators to phase- 
space functions can be inverted according to $\left\{H_{\alpha}(\hat{p}, \hat{q})\right\}_{\alpha}=\widehat{H}$. Here the mapping

$$
H \mapsto\{H(\hat{p}, \hat{q})\}_{\alpha}:=\int \frac{d p d q}{(2 \pi)^{d}} \mathrm{e}^{i(\hat{p}-p) \cdot q} H(p, \hat{q}+\alpha q)
$$

is a quantization or operator-ordering scheme [2] for phase-space functions $H$ which gives for $\alpha=0,1 / 2,1$ anti-standard ( $\hat{p}$ left of $\hat{q}$ ), Weyl-Wigner (totally symmetrized) and standard ( $\hat{p}$ right of $\hat{q}$ ) ordering, respectively. A nice illustration is already provided by the example of a standard Hamiltonian

$$
\begin{aligned}
\widehat{H} & =\frac{1}{2}(\hat{p}-a(\hat{q}))^{2}+v(\hat{q}) \\
H_{\alpha}(p, q) & =\frac{1}{2}(p-a(q))^{2}+i\left(\alpha-\frac{1}{2}\right)(\nabla \cdot a)(q)+v(q) .
\end{aligned}
$$

Note that the $\alpha$-symbol $H_{\alpha}$ is real for all Hermitian $\widehat{H}$ if and only if $\alpha=1 / 2$.

We associate with a given $\widehat{H}$ for each $\alpha$ the operator-valued function

$$
\widehat{R}_{\alpha}(t):=\int \frac{d p d q}{(2 \pi)^{d}} \mathrm{e}^{i(\hat{p}-p) \cdot q} \exp \left\{-t H_{\alpha}(p, \hat{q}+\alpha q)\right\}, \quad t \geq 0 .
$$

It provides a short-time approximation to the semigroup $\exp (-t \widehat{H})$ in the sense that

$$
\widehat{R}_{\alpha}(0)=\hat{1}, \quad \dot{\widehat{R}}_{\alpha}(0):=\left.\frac{d}{d t} \widehat{R}_{\alpha}(t)\right|_{t=0}=-\widehat{H} .
$$

The idea now is to represent $\exp (-t \widehat{H})$ for arbitrary $t \geq 0$ as $\left[\widehat{R}_{\alpha}\left(\frac{t}{n}\right)\right]^{n}$ in the limit $n \rightarrow \infty$ by writing

$$
\begin{aligned}
\exp \{-t \widehat{H}\}= & \exp \left\{t \dot{\widehat{R}}_{\alpha}(0)\right\}=\lim _{n \rightarrow \infty}\left[\widehat{R}_{\alpha}\left(\frac{t}{n}\right)\right]^{n} \\
= & \lim _{n \rightarrow \infty} \int \frac{d p^{(n)} d q^{(n)}}{(2 \pi)^{d}} \cdot \ldots \cdot \frac{d p^{(1)} d q^{(1)}}{(2 \pi)^{d}} \mathrm{e}^{-i q^{(n)} \cdot \hat{p}} \\
& \quad \times \exp \left\{\sum_{\nu=1}^{n}\left[i p^{(\nu)} \cdot\left(q^{(\nu)}-q^{(\nu-1)}\right)-\frac{t}{n} H_{\alpha}\left(p^{(\nu)}, \hat{q}+q^{(\nu, \alpha)}\right)\right]\right\}
\end{aligned}
$$

where $q^{(0)}:=0$ and the so-called $\alpha$-point between $q^{(\nu)}$ and $q^{(\nu-1)}$ is defined as $q^{(\nu, \alpha)}:=\alpha q^{(\nu)}+(1-\alpha) q^{(\nu-1)}$. It is customary and mnemonically convenient to use a continuum notation for the right-hand side so that

$$
\mathrm{e}^{-t \widehat{H}}=\int_{\alpha} \mathcal{D} \eta \mathcal{D} \xi \mathrm{e}^{-i \xi(t) \cdot \hat{p}} \exp \left\{\int_{0}^{t} d s\left[i \eta(s) \cdot \dot{\xi}(s)-H_{\alpha}(\eta(s), \hat{q}+\xi(s))\right]\right\}
$$

This expression is interpreted as a formal integral over all paths $s \mapsto(\eta(s), \xi(s))$ in phase-space $\mathbb{R}^{d} \times \mathbb{R}^{d}$ with $\xi(0)=0$. The subscript $\alpha$ attached to the integration sign reminds that it is essential to strictly use the $\alpha$-point discretization together with the $\alpha$-symbol [31, 30, 38]. Otherwise there is no guarantee that the phase-space path integral represents the given semigroup. Conversely, replacing $H_{\alpha}$ in (44) by a given classical Hamilton function $H$ leads to the semigroup generated by the $\alpha-$ ordered Hamilton operator $\{H(\hat{p}, \hat{q})\}_{\alpha}$. In case of the standard Hamiltonian (38) the 
momentum variables in (43) can be integrated out yielding a discretized expression for the Feynman-Kac- $\alpha$ formula (cf. (24) and (6)). Thereby one recognizes the well-known fact that the $\alpha$-point discretization underlies the definition of the $\alpha-$ stochastic integral [33 39, p. 136], which hence is in one-to-one correspondence with the $\alpha$-ordering scheme (see also [30]). This is reflected by the conversion formula

$$
\frac{1}{2}(\hat{p} g(\hat{q})+g(\hat{q}) \hat{p})=\{\hat{p} g(\hat{q})\}_{\alpha}+i\left(\frac{1}{2}-\alpha\right)(\nabla \cdot g)(\hat{q})
$$

similar to (同).

The basic ingredient for the validity of (43) is the generalized Lie-Trotter formula

$$
\lim _{n \rightarrow \infty}\left[\widehat{F}\left(\frac{t}{n}\right)\right]^{n}=\exp \{t \dot{\widehat{F}}(0)\} \quad \text { if } \quad \widehat{F}(0)=\hat{1} .
$$

It can easily be verified by a Taylor expansion if $\widehat{F}(t)$ acts on a finite-dimensional Hilbert space. Suitable technical assumptions for the extension to the infinitedimensional case are specified in [11], see also [15]. The (semigroup version of the) original Lie-Trotter formula (see, e.g. 42]) corresponds to the choice $\widehat{F}(t)=\mathrm{e}^{-t \widehat{A}} \mathrm{e}^{-t \widehat{B}}$ for operators $\widehat{A}$ and $\widehat{B}$ obeying suitable conditions. Nelson [36] was the first to use it explicitly in the context of path integration. Note that without the presence of the Wiener measure the application of the original Lie-Trotter formula with $\widehat{A}=\hat{p}^{2} / 2$ and $\widehat{B}=v(\hat{q})$ is of much less analytical value than the standard Feynman-Kac formula (for $a=0$ ). In fact, one can use the latter to prove norm-convergence in the former 23].

An interesting question is whether it is possible to construct a measure on a suitable set of paths in phase space in order to benefit from general integration theory also in the case of non-standard Hamiltonians. One possibility is offered by Wiener-regularized expressions in the spirit of [13]. Another one derives from (22] for Hamiltonians of the form $\widehat{H}=h_{+}(\hat{p}) h_{-}(\hat{q})+h_{-}(\hat{q}) h_{+}(\hat{p})$. Both methods may also be used for systems with other than canonical degrees of freedom, e.g. spin systems.

We want to thank the local organizing committee for making possible this stimulating conference. H. L. acknowledges support by the Heisenberg-Landau programme. B. B. and S. W. want to thank the Studienstiftung des deutschen Volkes for support.

\section{References}

[1] J. Adamowski, B. Gerlach, H. Leschke, General aspects of the functional-integral approach to the polaron and related systems, in: Polarons and excitons in polar semiconductors and ionic crystals, ed. by J. T. Devreese, F. Peeters, NATO ASI Series B Vol. 108, Plenum, New York 1984

[2] G. S. Agarwal, E. Wolf, Calculus for functions of non-commuting operators and general phase-space methods in quantum mechanics I-III, Phys. Rev. D 2, 2161-2186, 2187-2205, 2206-2225 (1970)

[3] M. Aizenman, B. Simon, Brownian motion and Harnack inequality for Schrödinger operators, Commun. Pure Appl. Math. 35, 209-273 (1982)

[4] A. L. Alimov, On the relationship between functional integrals and differential equations, Theor. Math. Phys. 11, 434-439 (1972). Russian original: Teor. Mat. Fiz. 11, 182-189 (1972)

[5] L. Arnold, Stochastic differential equations - Theory and applications, Wiley-Interscience, New York 1974

[6] R. Azencott, H. Doss, L'equation de Schrödinger quand $\hbar$ tend vers zero; une approche probabiliste, in: Stochastic aspects of classical and quantum systems, ed. by S. Albeverio, P. Combe, M. SirugueCollin, Lecture Notes in Mathematics 1109, Springer, Heidelberg 1985

[7] H. Bauer, Probability theory, de Gruyter, Berlin 1996 
[8] F. A. Berezin, Feynman path integrals in a phase space, Sov. Phys. Usp. 23, 763-788 (1980). Russian original: Usp. Fiz. Nauk 132, 497-548 (1980)

[9] K. Broderix, N. Heldt, H. Leschke, Aspects of Landau-level broadening using the path integral representation, Il Nuovo Cimento D 11, 241-259 (1989)

[10] K. Broderix, D. Hundertmark, H. Leschke, Continuity properties of Schrödinger semigroups with magnetic fields, preprint of the Universities Göttingen, Bochum and Erlangen-Nürnberg

[11] P. R. Chernoff, Note on product formulas for operator semigroups, J. Funct. Analysis 2, 238-242 (1968)

[12] H. L. Cycon, R. G. Froese, W. Kirsch, B. Simon, Schrödinger operators, Springer, Berlin 1987

[13] I. Daubechies, J. R. Klauder, Quantum-mechanical path integrals with Wiener measure for all polynomial Hamiltonians II, J. Math. Phys. 26, 2239-2256 (1985)

[14] J. S. Dowker, Path integrals and ordering rules, J. Math. Phys. 17, 1873-1874 (1976)

[15] P. Exner, Open quantum systems and Feynman integrals, Reidel, Dordrecht 1985, Appendix A.7-A.9

[16] R. P. Feynman, Space-time approach to non-relativistic quantum mechanics, Rev. Mod. Phys. 20, 367-387 (1948)

[17] R. P. Feynman, An operator calculus having applications in quantum electrodynamics, Phys. Rev. 84, 108-128 (1951), Appendices A-C

[18] W. Fischer, H. Leschke, P. Müller, The functional-analytic versus the functional-integral approach to quantum Hamiltonians: The one-dimensional hydrogen atom, J. Math. Phys. 36, 2313-2323 (1995)

[19] B. Gaveau, L. S. Schulman, Sensitive terms in the path integral: Ordering and stochastic options, J. Math. Phys. 30, 2019-2022 (1989)

[20] J. Glimm, A. Jaffe, Quantum physics, second ed., Springer, New York 1987, Appendix to part I

[21] Z. Haba, Semiclassical stochastic representation of the Feynman integral, J. Phys. A 27, 6457-6477 (1994)

[22] T. Hida, H.-H. Kuo, J. Potthoff, L. Streit, White noise - An infinite dimensional calculus, Kluwer, Dordrecht 1993

[23] T. Ichinose, S. Takanobu, Estimate of the difference between the Kac operator and the Schrödinger semigroup, preprint of the Kanazawa University, Japan; see also pp. 341-346 this volume

[24] M. Kac, On distributions of certain Wiener functionals, Trans. Amer. Math. Soc. 65, 1-13 (1949)

[25] M. Kac, On some connections between probability theory and differential and integral equations, in: Proceedings of the second Berkeley symposium on probability and statistics, ed. by J. Neyman, University of California, Berkeley 1951

[26] R. Z. Khas'minskii, On positive solutions of the equation $U u+V u=0$, Theory Probab. Appl. 4, 309-318 (1959). Russian original: Teor. Veroyatnost. i Primenen. 4, 332-341 (1959)

[27] E. A. Kochetov, S. P. Kuleshov, M. A. Smondyrev, Functional variational approach to polaron models, Sov. J. Part. Nucl. 13, 264-277 (1982). Russian original: Fiz. Elem. Chastits At. Yadra 13, 635-668 (1982)

[28] R. Kubo, Generalized cumulant expansion method, J. Phys. Soc. (Japan) 17, 1100-1120 (1962)

[29] S. Lang, Real and functional analysis, third ed., Springer, New York 1993

[30] F. Langouche, D. Roekaerts, E. Tirapegui, Functional integration and semiclassical expansion, Reidel, Dordrecht 1982

[31] H. Leschke, M. Schmutz, Operator orderings and functional formulations of quantum and stochastic dynamics, Z. Physik B 27, 85-94 (1977)

[32] P. Malliavin, Stochastic analysis, Springer, to appear

[33] T. P. McGarty, Stochastic systems and state estimation, Wiley-Interscience, New York 1974

[34] B. Mühlschlegel, Functional integrals and local many-body problems: Localized moments and small particles, in: Functional integration and its applications, ed. by A. M. Arthurs, Clarendon, Oxford 1975

[35] V. E. Nazaikinskii, V. E. Shatalov, B. Yu. Sternin, Methods of noncommutative analysis, de Gruyter, Berlin 1996

[36] E. Nelson, Feynman integrals and the Schrödinger equation, J. Math. Phys. 5, $332-343$ (1964)

[37] D. Nualart, The Malliavin calculus and related topics, Springer, New York 1995

[38] L. V. Prokhorov, Hamiltonian path integrals, Sov. J. Part. Nucl. 13, 456-482 (1982). Russian original: Fiz. Elem. Chastits At. Yadra 13, 1094-1156 (1982)

[39] D. Revuz, M. Yor, Continuous martingales and Brownian motion, second ed., Springer, Berlin 1994

[40] G. Roepstorff, Path integral approach to quantum physics, Springer, Berlin 1994

[41] S. M. Rytov, Yu. A. Kravtsov, V. I. Tatarskii, Principles of statistical radiophysics, Vol. 3, Springer, Berlin 1989

[42] B. Simon, Functional integration and quantum physics, Academic, New York 1979

[43] B. Simon, Schrödinger semigroups, Bull. Amer. Math. Soc. (N.S.) 3, 447-526 (1982), erratum: ibid. 11, 426 (1984)

[44] S. Weinberg, The quantum theory of fields, Vol. I, repr. with corrections, CUP, Cambridge 1996

[45] N. Wiener, Differential space, J. Mathematical and Physical Sci. 2, 131-174 (1923)

[46] Ya. B. Zeldovich, A. A. Ruzmaikin, D. D. Sokoloff, The almighty chance, World-Scientific, Singapore 1990, p. 90 